\title{
A Quasi-constant Envelope Multiplexing Technique for GNSS Signals
}

\author{
Tao Yan, Zuping Tang, Jiaolong Wei, Bo Qu and Zhihui Zhou \\ (School of Electronic Information and Communications, Huazhong University of \\ Science and Technology, Wuhan, 430074, China) \\ (E-mail: tang_zuping@mail.hust.edu.cn)
}

\begin{abstract}
A significant feature of the modernised Global Navigation Satellite System (GNSS) signals is that there are multiple signal components needing to be transmitted on a carrier frequency. How to combine these signal components into a constant envelope composite signal is a challenge. Existing constant envelope modulation techniques have some limitations, and are not effective enough. To solve this problem, we propose a quasi-constant-envelope multiplexing technique in this paper. The proposed method is based on numerical optimisation, and can work in two ways. The corresponding objective functions are provided. To verify the performance of the proposed method, we present three application examples. Results show that the first variation of our method can reach the same combining performance as Phase-Optimised Constant-Envelope Transmission (POCET). In the second variation, the combining efficiency can be pre-set. We can reach higher combining efficiency than POCET, and the envelope of the composite signal becomes quasi-constant. Furthermore, the inter-modulation signals in the final composite signal are adjustable. With the help of the proposed method, we can learn more details of the combining scheme than with POCET.
\end{abstract}

\section{KEYWORDS}
1. Quasi-constant envelope.
2. Combining efficiency. Satellite System (GNSS).
3. POCET.
4. Global Navigation

Submitted: 19 May 2014. Accepted: 27 January 2015. First published online: 5 March 2015.

1. INTRODUCTION. For the modernised Global Navigation Satellite System (GNSS) signals, there are more signal components needing to be transmitted on the same carrier frequency compared with traditional Global Positioning System (GPS) signals. However, in order to maximize efficiency of a nonlinear High-Power Amplifier (HPA), it is preferred that the HPA operates at saturation of its nonlinear region. To reduce Amplitude Modulation to Amplitude Modulation (AM/AM) and Amplitude Modulation to Phase Modulation (AM/PM) distortions, constant envelope modulation techniques are required (Dafesh and Cahn, 2009). Traditional constant envelope modulation techniques include Interplex (Butman and Timor, 1972), Majority Vote (MV), Inter-vote (Spilker and Orr, 1988) and Coherent Adaptive Subcarrier Modulation 
(CASM) (Dafesh et al., 1999). Among them, CASM is mathematically equivalent to Interplex (Fan et al., 2008). Inter-vote is a synthetic technique of MV and Interplex. Nevertheless, the above techniques have some limitations. For example, when the signal component with the highest power is orthogonal with other signal components, CASM reaches the best performance (Dafesh et al., 1999). Majority vote can combine an odd number of signals, and works best when every signal component has equal power (Spilker and Orr, 1988). Inter-vote has the advantages of both Interplex and $\mathrm{MV}$, but the number of signal components is at least five.

A more general technique referred to as Phase-Optimised Constant-Envelope Transmission (POCET) was proposed by Dafesh and Cahn (2009). This can combine $N$ binary Pseudo Random Noise (PRN) code signals into a constant envelope composite signal by a numerical optimisation process. This final composite signal can be seen as a phase modulation signal. On this basis, POCET is further used to combine GNSS signals at different carrier frequencies (Dafesh and Cahn, 2011), which is implemented by phase rotation. In general, POCET can reach the highest combining efficiency by optimising the phases. Nevertheless, Dafesh and Cahn do not provide the analytical expression of POCET. The composite signal of the POCET method cannot be written into an expression of the useful signal components and InterModulation (IM) signals (Zhang et al., 2012a). This problem has been solved by Zhang et al. (2012b). They derive the analytical expression of POCET by referring to a classical problem in digital logic. Although the POCET method can be applied for most cases, it still has the following drawbacks. Firstly, POCET is only applicable for unrelated binary PRN signals (Zhang et al., 2011). In other words, POCET cannot deal with the case when some signal components are related. Secondly, it is not possible for POCET to suppress some undesired IM product signals. Finally, POCET cannot provide any information about how to achieve slightly better combining efficiency.

The aim of this paper is to propose a more effective multiplexing technique for GNSS signals, from which we can know more details of the combining. This proposed method is based on numerical optimisation. In contrast to POCET, we optimise these complex coefficients of the useful signal components and IM signals. This method can work in two ways. In the first way, the multiplexing performance of our method is the same as POCET. Then, the IM signals in the final composite signal are controllable. In the second way, the combining efficiency is adjustable. Namely, we can set the combining efficiency before the optimisation process. We can even obtain higher combining efficiency than POCET. In this case, the envelope of the final composite signal becomes quasi-constant.

The remainder of this paper is organised as follows. In Section 2, we recall the POCET method, and describe the proposed quasi-constant-envelope multiplexing method. Then our method is applied for the GPS L1 signals in the first way in Section 3. In Section 4, the multiplexing example of non-independent signals is discussed. In Section 5, the Alternative Binary Offset Carrier (AltBOC) -like modulation schemes with $85 \%$ and $86 \%$ combining efficiency are presented in the second way. Conclusions are summarised in the last section.

2. DESCRIPTION OF THE PROPOSED METHOD. We first briefly review the POCET method (Dafesh and Cahn 2009; Cahn and Defesh 2011). The POCET 
method can combine $N$ binary PRN code signals into a constant envelope signal. The final composite signal is equivalent to a phase shift-keying signal.

2.1. POCET method. For the $N$ binary PRN code signals, there are a possible $2^{N}$ different signal vectors. Each signal vector corresponds to a phase value $\theta_{k}, k=0,1, \ldots$, $2^{N}-1$. By optimising these $2^{N}$ phase values, the designed power and phase constraints between signal components are met.

In order to derive the correlation output of every PRN code signal, there are two assumptions made in the POCET method (Zhang et al., 2011). Firstly, the $N$ binary PRN code signals are uncorrelated. Secondly, every PRN code is completely random. Thus, the $2^{N}$ phase values occur with equal probability. Then the average correlation for the $n$th signal component is expressed as

$$
\operatorname{corr}_{n}=\frac{A}{2^{N}} \sum_{k=0}^{2^{N}-1} b_{n}(k) e^{j \theta_{k}}
$$

where $A$ is the envelope of the composite signal. $b_{n}(k)= \pm 1$ is the $n$th signal component in the $k$ th signal vector, and $j=\sqrt{-1}$ is the imaginary unit. In order to maximise the combining efficiency, the objective of POCET is to minimise the envelope $A$ subject to the power and phase constraints.

The power constraints are given by

$$
P_{d n}=\left|\operatorname{corr}_{n}(\boldsymbol{\theta})\right|^{2}=\left|\frac{A}{2^{N}} \sum_{k=0}^{2^{N}-1} b_{n}(k) e^{j \theta_{k}}\right|^{2}
$$

where $P_{d n}$ is the desired power level of the $n$th signal component. When the relative phase difference between signal $n$ and signal $l$ is $\Delta \phi_{n l}$, the phase constraints between these two signal components are

$$
\begin{aligned}
& \operatorname{Im}\left\{e^{-j \Delta \phi_{n l} l} \operatorname{corr}_{n}(\boldsymbol{\theta}) \operatorname{corr}_{l}(\boldsymbol{\theta})^{*}\right\}=0 \\
& \operatorname{Re}\left\{e^{\left.-j \Delta \phi_{n l} l \operatorname{corr}_{n}(\boldsymbol{\theta}) \operatorname{corr}_{l}(\boldsymbol{\theta})^{*}\right\}>0}\right.
\end{aligned}
$$

By using the penalty function method, the constrained optimisation problem for POCET is converted into the following unconstrained optimisation problem, i.e.

$$
\min \left\{\begin{aligned}
F(\theta)= & A^{2}+\mu_{a} \sum_{n=1}^{N}\left(\left|\operatorname{corr}_{n}(\boldsymbol{\theta})\right|-\operatorname{corr}_{n}\right)^{2} \\
& +\mu_{b} \sum_{n=1}^{N-1} \sum_{l=n+1}^{N} \operatorname{Im}\left\{e^{-j \Delta \phi_{n l}} \operatorname{corr}_{n}(\boldsymbol{\theta}) \operatorname{corr}_{l}(\boldsymbol{\theta})^{*}\right\}^{2}
\end{aligned}\right\}
$$

where $\operatorname{corrd}_{n}=\sqrt{P_{d n}}, \mu_{a}$ and $\mu_{b}$ are positive penalty factors. We note that the second part of Equation (3) is not considered in Equation (4), which may result in a $180^{\circ}$ ambiguity. Of course, this ambiguity can be solved by analysing the phase relationship calculated from the optimum solution (Zhang et al., 2011). POCET requires that the phase table is symmetrical. Namely, when two signal vectors are complementary, the difference of the corresponding phase values is $180^{\circ}$. Thus, $\theta_{k}=\theta_{2^{N}-1-k}+\pi$. $\theta_{0}$ can be set to 0 . Therefore, there are only $2^{N-1}-1$ unknown phase values.

2.2. The proposed method. According to the results of Zhang et al. (2012b), we know that for $N$ unrelated binary PRN signals $\left\{s_{1}(t), s_{2}(t), \cdots, s_{N}(t)\right\}$, the general 
expression of constant envelope composite signal is expressed as

$$
\begin{aligned}
s(t)= & c_{0}+\sum_{n=1}^{N} c_{n} e^{j \theta_{n}} s_{n}(t)+\sum_{n_{1}=1}^{N-1} \sum_{n_{2}=n_{1}+1}^{N} c_{n_{1}, n_{2}} e^{j \theta_{n_{1}, n_{2}}} s_{n_{1}}(t) s_{n_{2}}(t) \\
& +\sum_{n_{1}=1}^{N-2} \sum_{n_{2}=n_{1}+1}^{N-1} \sum_{n_{3}=n_{2}+1}^{N} c_{n_{1}, n_{2}, n_{3}} e^{j \theta_{n 1, n 2, n 3}} s_{n_{1}}(t) s_{n_{2}}(t) s_{n_{3}}(t) \\
& +\cdots+c_{1,2, \cdots, N} s_{1}(t) s_{2}(t) \cdots s_{N}(t),
\end{aligned}
$$

The composite signal $s(t)$ may include an un-modulated carrier component, $N$ desired single signal components and $2^{N}-1-N$ IM signals. The $2^{N}$ complex coefficients are denoted as $A_{0} e^{j \theta_{0}}, A_{1} e^{j \theta_{1}}, \cdots, A_{2^{N}-1} e^{j \theta_{2^{N}-1}}$, then Equation (5) can be written as:

$$
s(t)=\mathbf{S}_{1 \times 2^{N}} \cdot \mathbf{C}
$$

where $\mathbf{S}_{1 \times 2^{N}}$ is a $1 \times 2^{N}$ row vector, $\mathbf{C}$ is a $2^{N} \times 1$ column vector composed of all these complex coefficients. They are given by

$$
\begin{aligned}
& \mathbf{S}_{1 \times 2^{N}}=\left[\begin{array}{llllllllll}
1 & s_{1} & \cdots & s_{N} & s_{1} s_{2} & \cdots & s_{N-1} s_{N} & \cdots & \prod_{n=2}^{N} s_{n} & \prod_{n=1}^{N} s_{n}
\end{array}\right] \\
& \mathbf{C}=\left[\begin{array}{l}
A_{0} e^{j \theta_{0}}, A_{1} e^{j \theta_{1}}, \cdots, A_{N} e^{j \theta_{N}}, A_{N+1} e^{j \theta_{N+1}}, \cdots, \\
A_{N+N(N-1) / 2} e^{j \theta_{N+N(N-1) / 2}}, \cdots, A_{2^{N}-2} e^{j \theta_{2}-2}, A_{2^{N}-1} e^{j \theta_{2}-1}
\end{array}\right]^{T}
\end{aligned}
$$

Since every binary PRN signal has two values, 1 or $-1, \mathbf{S}_{1 \times 2^{N}}$ has $2^{N}$ different states. When taking these states into account, we obtain a $2^{N} \times 2^{N}$ matrix $\mathbf{S}$ derived from vector $\mathbf{S}_{1 \times 2^{N}}$. For a given $N, \mathbf{S}$ is determined. For example, when $N=3, \mathbf{S}_{1 \times 2^{N}}$ and $\mathbf{S}$ are

$$
\begin{aligned}
& \mathbf{S}_{1 \times 2^{N}}=\left[\begin{array}{lllrrrrr}
1 & s_{1} & s_{2} & s_{3} & s_{1} s_{2} & s_{1} s_{3} & s_{2} s_{3} & s_{1} s_{2} s_{3}
\end{array}\right], \\
& \mathbf{S}=\left[\begin{array}{rrrrrrrr}
1 & 1 & 1 & 1 & 1 & 1 & 1 & 1 \\
1 & 1 & 1 & -1 & 1 & -1 & -1 & -1 \\
1 & 1 & -1 & 1 & -1 & 1 & -1 & -1 \\
1 & 1 & -1 & -1 & -1 & -1 & 1 & 1 \\
1 & -1 & 1 & 1 & -1 & -1 & 1 & -1 \\
1 & -1 & 1 & -1 & -1 & 1 & -1 & 1 \\
1 & -1 & -1 & 1 & 1 & -1 & -1 & 1 \\
1 & -1 & -1 & -1 & 1 & 1 & 1 & -1
\end{array}\right]
\end{aligned}
$$

respectively. According to Equation (6), we can see that $s(t)$ has $2^{N}$ possible values. These values are expressed as a $2^{N} \times 1$ column vector $\mathbf{s}$, i.e.

$$
\mathbf{s}=\mathbf{S C}=\left[\begin{array}{c}
f_{1}\left(A_{0}, \cdots, A_{2^{N}-1}, \theta_{0}, \cdots, \theta_{2^{N}-1}\right) \\
\cdots \\
f_{N}\left(A_{0}, \cdots, A_{2^{N}-1}, \theta_{0}, \cdots, \theta_{2^{N}-1}\right) \\
\cdots \\
f_{2^{N}}\left(A_{0}, \cdots, A_{2^{N}-1}, \theta_{0}, \cdots, \theta_{2^{N}-1}\right)
\end{array}\right]=\mathbf{S C}_{\text {real }}+j \mathbf{S C}_{\text {imag }}
$$


where

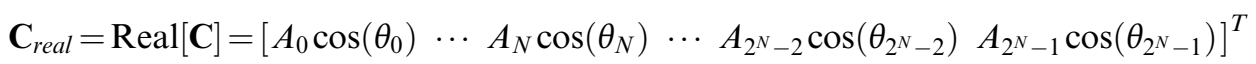

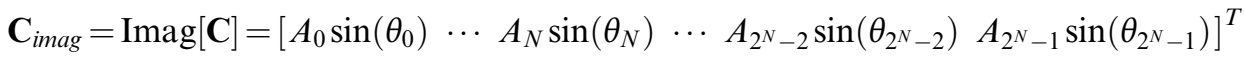

Each element in $\mathbf{s}$ is a function of all the complex coefficients. Our method is to directly optimise these complex coefficients in $\mathbf{C}$ to force the amplitude of every element in $\mathbf{s}$ to be the same. Generally speaking, the power relationship between signal components is designed. This is why POCET is subject to the power constraints. In our method, however, the designed power relationship is easily satisfied. Only the following equation is established, i.e.

$$
A_{1}^{2}: A_{2}^{2}: \cdots: A_{N}^{2}=P_{1}: P_{2}: \cdots: P_{N}
$$

where the right part of Equation (10) is the designed power allocation ratio. Without loss of generality, we set $A_{1}=1$, then

$$
A_{n}=\sqrt{P_{n} / P_{1}}, n=1,2, \cdots, N
$$

Clearly, if we expect that some IM product signals do not exist in the final composite signal, we can directly set the corresponding coefficients to be zero.

In most cases, the phase relation between signal components has been designed. In fact, when there are phase constraints, we can pre-set the following phase relation:

$$
\theta_{n}=\theta_{l}+\Delta \theta_{n, l}, n>l
$$

where $\Delta \theta_{n, l}$ is the designed phase difference between signal $n$ and signal $l$. For example, we have the phase constraints $\Delta \theta_{2,1}$ and $\Delta \theta_{4,3}$, then we pre-set the phase relation following the order,

$$
\theta_{2}=\theta_{1}+\Delta \theta_{1,2}, \theta_{4}=\theta_{3}+\Delta \theta_{3,4}
$$

Evidently, $\theta_{2}$ and $\theta_{4}$ are determined by $\theta_{1}$ and $\theta_{3}$ respectively. We can see that there is no $180^{\circ}$ phase ambiguity in our method. Thus, we do not need the step to solve the ambiguity. When the operation is performed, an additional advantage is that the number of the variables decreases.

In order to keep the envelope of the composite signal $s(t)$ constant, every element in $s$ should have the same amplitude, i.e.

$$
\left|f_{0}\right|=\left|f_{1}\right|=\cdots=\left|f_{2^{N}-1}\right|=A_{\text {envelope }},
$$

where $A_{\text {envelope }}$ is the envelope value of the composite signal $s(t)$. When Equation (14) is true, we have the following constant envelope constraint:

$$
A_{\text {envelope }}=\sqrt{\frac{\|\mathbf{s}\|^{2}}{2^{N}}},|\mathbf{s}|-A_{\text {envelope }}=\mathbf{0}_{2^{N} \times 1},
$$

where $\|\mathbf{s}\|$ is the Euclidean norm of vector $s$, it can be calculated by $\|\mathbf{s}\|=\sqrt{\mathbf{s}^{H} \mathbf{s}} . \mathbf{s}^{H}$ is the conjugate transpose of $\mathbf{s} .\|\mathbf{s}\|$ is the absolute value of vector $\mathbf{s} . \mathbf{0}_{2^{N} \times 1}$ is a $2^{N} \times 1$ zero 
vector. Equation (5) is equivalent to the following equation, i.e.

$$
\left\||\mathbf{s}|-\sqrt{\frac{\|\mathbf{s}\|^{2}}{2^{N}}}\right\|=0 .
$$

If the $N$ binary PRN signals are completely unrelated, substituting Equation (9) into Equation (15), we derive that

$$
\begin{aligned}
A_{\text {envelope }}^{2}=\frac{\|\mathbf{S}\|^{2}}{2^{N}} & =\frac{1}{2^{N}}\left(\left(\mathbf{S C}_{\text {real }}\right)^{T}\left(\mathbf{S C}_{\text {real }}\right)+\left(\mathbf{S C}_{\text {imag }}\right)^{T}\left(\mathbf{S C}_{\text {imag }}\right)\right) \\
& =\frac{1}{2^{N}}\left(\mathbf{C}_{\text {real }}^{T}\left(\mathbf{S}^{T} \mathbf{S}\right) \mathbf{C}_{\text {real }}+\mathbf{C}_{\text {imag }}^{T}\left(\mathbf{S}^{T} \mathbf{S}\right) \mathbf{C}_{\text {imag }}\right)
\end{aligned}
$$

In this case, $\mathbf{S}^{T} \mathbf{S} / 2^{N}=\mathbf{I}_{2^{N} \times 2^{N}}$ (Zhang et al., 2012a), we have

$$
A_{\text {envelope }}^{2}=\mathbf{C}_{\text {real }}^{T} \mathbf{C}_{\text {real }}+\mathbf{C}_{\text {imag }}^{T} \mathbf{C}_{\text {imag }}=\sum_{n=0}^{2^{N}-1} A_{n}^{2}
$$

It is interesting that the envelope value $A_{\text {envelope }}$ is only determined by the $2^{N}$ amplitude values of these complex coefficients, and is independent of the $2^{N}$ phase values.

The power of the desired signals is given by Equation (11). Then the combining efficiency is expressed as

$$
\eta=\frac{\sum_{n=1}^{N} A_{n}^{2}}{A_{\text {envelope }}^{2}}=\frac{\sum_{n=1}^{N} A_{n}^{2}}{\frac{\|\mathbf{s}\|^{2}}{2^{N}}}=\frac{2^{N} \sum_{n=1}^{N} A_{n}^{2}}{\|\mathbf{s}\|^{2}}
$$

To maximise the combining efficiency, we should minimise $A_{\text {envelope }}^{2}$ by optimising these complex coefficients. At the same time, Equation (16) has to be satisfied. This is a constrained nonlinear optimisation problem. Similar to the POCET method, this optimisation problem can be converted into the following unconstrained optimisation problem:

$$
\underset{\left\{A_{n}, \theta_{n}\right\}}{\arg \min }\left(\frac{\|\mathbf{s}\|^{2}}{2^{N}}+\mu_{a} \||\mathbf{s}|-\left.\sqrt{\frac{\|\mathbf{s}\|^{2}}{2^{N}}}\right|^{2}\right),
$$

where $\mu_{a}$ is the positive penalty factor. To find out the optimal numerical solution of Equation (20), we adopt the search strategy from Dafesh and Cahn (2009). The specific optimisation process is not discussed here. The number of variables $\left\{A_{n}\right\}$ is $2^{N}$, and the number of variables $\left\{\theta_{n}\right\}$ is also $2^{N}$. So the total number of the variables is $2^{N+1}$. However, due to Equation (11), the number of the variables to be determined is $2^{N+1}-N$. When Equation (12) is used, the number of the variables would be further reduced.

Equation (20) is the final objective function, in which only the constant envelope constraint needs to be considered. Its solution can strictly meet the designed power and phase constraints. Moreover, from Section 3, we will see that the combining 
efficiency is the same as the POCET technique. Compared with POCET, the main advantage of our method is that we can easily suppress some undesired IM signals in the composite signal.

In contrast to the POCET method, our method can work in a second way. We can pre-set the combining efficiency $\eta_{\text {set }}$ before the optimisation process. By exploiting $\eta_{\text {set }}$, the pre-set envelope value $A_{\text {set }}$ is

$$
A_{\text {set }}=\sqrt{\frac{\sum_{n=1}^{N} A_{n}^{2}}{\eta_{\text {set }}}} .
$$

The constant envelope constraint is simplified correspondingly as

$$
A_{\text {set }}=\sqrt{\frac{\|\mathbf{s}\|^{2}}{2^{N}}},\left\||\mathbf{s}|-A_{\text {set }}\right\|=0
$$

The objective function in the second way is written as

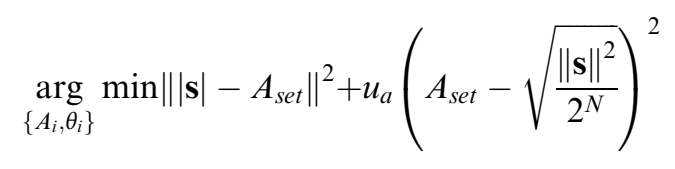

In the first method, when the power constraints and phase constraints of the signal components are given, the optimal numerical solution can be obtained by solving Equation (20). We denote the combining efficiency as $\eta_{\text {opt }}$. In the second method, if the pre-set combining efficiency $\eta_{\text {set }} \leq \eta_{\text {opt }}$, the optimal numerical solution of Equation (23) can keep the envelope of $s(t)$ constant. If the pre-set combining efficiency $\eta_{\text {set }}>\eta_{\text {opt }}$, the envelope of $s(t)$ would become quasi-constant. The optimal numerical solution in this case can ensure that the changes in the envelopes of $s(t)$ have the least root mean square error. Thus we call our method a quasi-constant envelope multiplexing technique. With the help of the second method, we can learn more details of the combining.

3. THE APPLICATION OF THE FIRST METHOD. The example is about the multiplexing scheme of GPS L1. There are four signal components. They are L1C/ A, L1P(Y), L1C $C_{\mathrm{P}}$ and $\mathrm{L} 1 \mathrm{C}_{\mathrm{D}}$ respectively. We denote them as $s_{1}, s_{2}, s_{3}$ and $s_{4}$. The normalised power of the four signal components are $0 \mathrm{dBW},-3 \mathrm{dBW}, 0 \cdot 25 \mathrm{dBW}$ and $-4 \cdot 5 \mathrm{dBW}$ respectively (Dafesh and Cahn, 2009). The phase difference between $s_{2}$ and $s_{1}$ is $\theta_{2,1}=90^{\circ}$. According to Equations (11) and (13), we set

$$
A_{1}=1, A_{2}=0.708, A_{3}=1.029, A_{4}=0.596, \theta_{2}=\theta_{1}+90^{\circ}
$$


In this example, $N=4$. The $\mathbf{S}_{1 \times 2^{N}}$ and $\mathbf{S}$ are expressed as

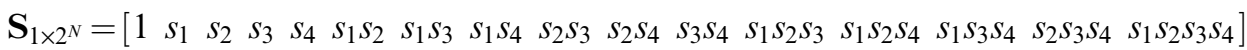

$$
\mathbf{S}=\left[\begin{array}{rrrrrrrrrrrrrrrr}
1 & 1 & 1 & 1 & 1 & 1 & 1 & 1 & 1 & 1 & 1 & 1 & 1 & 1 & 1 & 1 \\
1 & 1 & 1 & 1 & -1 & 1 & 1 & -1 & 1 & -1 & -1 & 1 & -1 & -1 & -1 & -1 \\
1 & 1 & 1 & -1 & 1 & 1 & -1 & 1 & -1 & 1 & -1 & -1 & 1 & -1 & -1 & 1 \\
1 & 1 & 1 & -1 & -1 & 1 & -1 & -1 & -1 & -1 & 1 & -1 & -1 & 1 & 1 & 1 \\
1 & 1 & -1 & 1 & 1 & -1 & 1 & 1 & -1 & -1 & 1 & -1 & -1 & 1 & -1 & -1 \\
1 & 1 & -1 & 1 & -1 & -1 & 1 & -1 & -1 & 1 & -1 & -1 & 1 & -1 & 1 & 1 \\
1 & 1 & -1 & -1 & 1 & -1 & -1 & 1 & 1 & -1 & -1 & 1 & -1 & -1 & 1 & 1 \\
1 & 1 & -1 & -1 & -1 & -1 & -1 & -1 & 1 & 1 & 1 & 1 & 1 & 1 & -1 & -1 \\
1 & -1 & 1 & 1 & 1 & -1 & -1 & -1 & 1 & 1 & 1 & -1 & -1 & -1 & 1 & -1 \\
1 & -1 & 1 & 1 & -1 & -1 & -1 & 1 & 1 & -1 & -1 & -1 & 1 & 1 & -1 & 1 \\
1 & -1 & 1 & -1 & 1 & -1 & 1 & -1 & -1 & 1 & -1 & 1 & -1 & 1 & -1 & 1 \\
1 & -1 & 1 & -1 & -1 & -1 & 1 & 1 & -1 & -1 & 1 & 1 & 1 & -1 & 1 & -1 \\
1 & -1 & -1 & 1 & 1 & 1 & -1 & -1 & -1 & -1 & 1 & 1 & 1 & -1 & -1 & 1 \\
1 & -1 & -1 & 1 & -1 & 1 & -1 & 1 & -1 & 1 & -1 & 1 & -1 & 1 & 1 & -1 \\
1 & -1 & -1 & -1 & 1 & 1 & 1 & -1 & 1 & -1 & -1 & -1 & 1 & 1 & 1 & -1 \\
1 & -1 & -1 & -1 & -1 & 1 & 1 & 1 & 1 & 1 & 1 & -1 & -1 & -1 & -1 & 1
\end{array}\right]
$$

By exploiting the objective function Equation (20), we obtain the optimal complex coefficients, which are listed in Table 1 . Without loss of generality, we set $\theta_{1}=0$. Then the composite signal $s(t)$ is expressed as

$$
\begin{aligned}
s(t)= & s_{1}+j 0.708 s_{2}-j 1.029 s_{3}-j 0.596 s_{4}+0.395 s_{1} s_{2} s_{3} \\
& -0.053 s_{1} s_{2} s_{4}-0.209 s_{1} s_{3} s_{4}-j 0.541 s_{2} s_{3} s_{4}
\end{aligned}
$$

Based on Equation (9), we can calculate the vector $s$. Every element in $s$ has the same amplitude. The phase angles of all the elements in $s$ can form the phase look-up table. Results are listed in Table 2.

The envelope value $A_{\text {envelope }}$ of Equation (26) is 1.847. According to Equation (19), we calculate the combining efficiency, which is $85 \cdot 47 \%$. The corresponding combining loss is $-0.68 \mathrm{~dB}$, which is the same as POCET (Dafesh and Cahn, 2009). This example verifies the correctness of our method in the first way. It shows that the combining efficiency of the first way is equivalent to the POCET method.

4. THE MULTIPLEXING OF NON-INDEPENDENT SIGNALS. Our method can also be used to combine non-independent signals to a certain extent. Namely, some signals are related. We take the multiplexing scheme of Galileo E1 signals as an example to demonstrate this. There are an Open Service (OS) signal and a Public Regulated Service (PRS) signal at the Galileo E1 frequency. The PRS signal is BOCc $(15,2 \cdot 5)$ modulation, denoted as $s_{P R S}(t)$. The OS signal is $\operatorname{CBOC}(6,1,1 / 11)$ modulation, it includes a data component $\left(\mathrm{CBOC}^{+}\right)$and a pilot component 
Table 1. The optimal complex coefficients for GPS L1 signals.

\begin{tabular}{ccccccccc}
\hline & 1 & $s_{1}$ & $s_{2}$ & $s_{3}$ & $s_{4}$ & $s_{1} s_{2}$ & $s_{1} s_{3}$ & $s_{1} s_{4}$ \\
\hline$A_{i}$ & $0 \cdot 0$ & 1 & $0 \cdot 708$ & $1 \cdot 029$ & $0 \cdot 596$ & $0 \cdot 0$ & $0 \cdot 0$ & $0 \cdot 0$ \\
$\theta_{i}$ & $10 \cdot 4$ & 0 & 90 & $-90 \cdot 0$ & $-90 \cdot 0$ & $-83 \cdot 1$ & $168 \cdot 0$ & $40 \cdot 3$ \\
& $s_{2} s_{3}$ & $s_{2} s_{4}$ & $s_{3} s_{4}$ & $s_{1} s_{2} s_{3}$ & $s_{1} s_{2} s_{4}$ & $s_{1} s_{3} s_{4}$ & $s_{2} s_{3} s_{4}$ & $s_{1} s_{2} s_{3} s_{4}$ \\
$A_{i}$ & $0 \cdot 0$ & $0 \cdot 0$ & $0 \cdot 0$ & $0 \cdot 395$ & $0 \cdot 053$ & $0 \cdot 209$ & $0 \cdot 541$ & $0 \cdot 0$ \\
$\theta_{i}$ & $-155 \cdot 5$ & $13 \cdot 0$ & $-171 \cdot 1$ & $0 \cdot 0$ & $180 \cdot 0$ & $180 \cdot 0$ & $-90 \cdot 0$ & $104 \cdot 1$ \\
\hline
\end{tabular}

Table 2. The corresponding phase look-up table.

\begin{tabular}{lrrrr}
\hline$s_{1}$ & $s_{2}$ & $s_{3}$ & $s_{4}$ & Phase angle (degrees) \\
\hline 1 & 1 & 1 & 1 & $-52 \cdot 1$ \\
1 & 1 & 1 & -1 & $26 \cdot 2$ \\
1 & 1 & -1 & 1 & $65 \cdot 7$ \\
1 & 1 & -1 & -1 & $75 \cdot 9$ \\
1 & -1 & 1 & 1 & $-75 \cdot 9$ \\
1 & -1 & 1 & -1 & $65 \cdot 7$ \\
1 & -1 & -1 & 1 & $-26 \cdot 2$ \\
1 & -1 & -1 & -1 & $52 \cdot 1$ \\
-1 & 1 & 1 & 1 & $-127 \cdot 9$ \\
-1 & 1 & 1 & -1 & $153 \cdot 8$ \\
-1 & 1 & -1 & 1 & $114 \cdot 3$ \\
-1 & 1 & -1 & -1 & $104 \cdot 1$ \\
-1 & -1 & 1 & 1 & $-104 \cdot 1$ \\
-1 & -1 & 1 & 1 & $-114 \cdot 3$ \\
-1 & -1 & -1 & -1 & $-153 \cdot 8$ \\
-1 & -1 & -1 & & $127 \cdot 9$ \\
\hline
\end{tabular}

$\left(\mathrm{CBOC}^{-}\right)$. They are expressed as

$$
\begin{aligned}
& s_{E 1-D}(t)=c_{E 1-D}(t)\left(s c_{B O C(1,1)} \cos \theta_{1}+s c_{B O C(6,1)} \cos \theta_{2}\right), \\
& s_{E 1-D}(t)=c_{E 1-D}(t)\left(s c_{B O C(1,1)} \cos \theta_{1}-s c_{B O C(6,1)} \cos \theta_{2}\right) .
\end{aligned}
$$

respectively, where $c_{E 1-D}(t)$ and $c_{E 1-P}(t)$ are the PRN codes of the data component and pilot component respectively. The constant envelope multiplexing scheme is Interplex (Hein et al., 2005). The expression of the composite signal is:

$$
s(t)=\sqrt{2 P}\left[\begin{array}{c}
\frac{c_{E 1-D}(t)}{2}\left(s c_{B O C(1,1)}(t) \cos \theta_{1}+s c_{B O C(6,1)}(t) \cos \theta_{2}\right)+ \\
\frac{c_{E 1-P}(t)}{2}\left(s c_{B O C(1,1)}(t) \cos \theta_{1}-s c_{B O C(6,1)}(t) \cos \theta_{2}\right)+ \\
j\left(s_{P R S}(t) \cdot \frac{\sin \theta_{1}+\sin \theta_{2}}{2}-s_{P R S}(t) c_{E 1-D}(t) c_{E 1-P}(t) \cdot \frac{\sin \theta_{2}-\sin \theta_{1}}{2}\right)
\end{array}\right] .
$$

When $\cos \theta_{1}=\sqrt{10 / 11}, \cos \theta_{2}=\sqrt{1 / 11}$, the power ratio of $s_{E 1-D}(t), s_{E 1-P}(t), s_{P R S}(t)$ and $s_{\mathrm{IM}}(t)$ is $1: 1: 1 \cdot 575: 0 \cdot 425$. The corresponding combining efficiency is $89 \cdot 37 \%$.

Now we try to obtain the multiplexing scheme using our method. Because the CBOC modulation has four values, our method cannot be applied directly to this 
case. Fortunately, the data component of the OS signal can be seen as the sum of two binary signals. The pilot component of the OS signal can be seen as the difference of two binary signals. Therefore, the problem can be converted into the multiplexing of five binary signals. The five signals are

$$
\begin{gathered}
s_{1}=c_{E 1-D}(t) s c_{B O C(6,1)}(t), s_{2}=c_{E 1-D}(t) s c_{B O C(1,1)}(t) \\
s_{3}=c_{E 1-P}(t) s c_{B O C(6,1)}(t), s_{4}=c_{E 1-P}(t) s c_{B O C(1,1)}(t) \\
s_{5}=s_{P R S}(t) .
\end{gathered}
$$

Their power ratio is 1:10:1:10:17·325. According to Equations (11) and (13), we set

$$
\begin{gathered}
A_{1}=A_{3}=1, A_{2}=A_{4}=\sqrt{10}=3.162, A_{5}=4.162, \\
\theta_{4}=\theta_{2}=\theta_{1}, \theta_{3}=\theta_{1}+180^{\circ}, \theta_{5}=\theta_{1}+90^{\circ} .
\end{gathered}
$$

There are 32 different possible states for five binary signals. However, in this case, $s_{1}$ and $s_{2}$ are related, $s_{3}$ and $s_{4}$ are related. Moreover, $s_{1} \cdot s_{3}=c_{E 1-D}(t) \cdot c_{E 1-P}(t)=s_{2}$. $s_{4}$. Thus, there are only 16 different states. The matrix $\mathbf{S}$ is a $16 \times 32$ matrix here. Vector $\mathbf{s}$ in Equation (20) becomes a $16 \times 1$ vector. We do not expect that the following IM signals exist in the composite signal, because

$$
\begin{gathered}
s_{1} s_{2}=s_{3} s_{4}=s c_{B O C(1,1)}(t) s c_{B O C(6,1)}(t), \\
s_{1} s_{2} s_{3}=s_{4}, s_{1} s_{2} s_{4}=s_{3}, s_{1} s_{3} s_{4}=s_{2}, s_{2} s_{3} s_{4}=s_{1}, \\
s_{1} s_{2} s_{5}=s_{3} s_{4} s_{5}=s_{5} s c_{B O C(6,1)}(t) s c_{B O C(1,1)}(t), \\
s_{1} s_{2} s_{3} s_{4}=1, s_{1} s_{2} s_{3} s_{4} s_{5}=s_{5} .
\end{gathered}
$$

Thus we pre-set the corresponding coefficients to be zero before the optimisation process, i.e.

$$
A_{6}=A_{13}=A_{16}=A_{17}=A_{18}=A_{19}=A_{22}=A_{25}=A_{26}=A_{31}=0
$$

At the same time, the un-modulated carrier component is also undesired, namely $A_{0}=0$.

By exploiting the objective function Equation (20), we obtain the optimal complex coefficients, which are listed in Table 3. The corresponding phase look-up table is listed in Table 4 . When the five signals are multiplexed by using these coefficients, combining efficiency is also $89.37 \%$, which is the same as Equation (28). The final composite signal is

$$
\begin{aligned}
& s(t)=s_{1}+3.162 s_{2}-s_{3}+3.162 s_{4}+j 4.162 s_{5}+0.634 e^{-j \pi \frac{97.1}{180}} s_{1} s_{3}+2.038 e^{-j \pi \frac{112.1}{180}} s_{1} s_{4} \\
& +1.25 e^{-j \pi \frac{55.5}{180}} s_{1} s_{5}+2.238 e^{j \pi \frac{67.9}{180}} s_{2} s_{3}+0.634 e^{j \pi \frac{82.9}{180}} s_{2} s_{4}+1.209 e^{-j \pi \frac{83.9}{180}} s_{1} s_{3} s_{5} \\
& +0.438 e^{j \pi \frac{144.0}{180}} s_{1} s_{4} S_{5}+0.438 e^{-j \pi \frac{36.0}{180}} s_{2} s_{3} s_{5}+0.968 e^{-j \pi \frac{97.6}{180}} s_{2} s_{4} s_{5}+1.25 e^{j \pi \frac{124.5}{180}} s_{2} s_{3} s_{4} s_{5}
\end{aligned}
$$


Table 3. The optimal complex coefficients for Galileo E1 signals.

\begin{tabular}{lllllllll}
\hline & 1 & $s_{1}$ & $s_{2}$ & $s_{3}$ & $s_{4}$ & $s_{5}$ & $s_{1} s_{2}$ & $s_{1} s_{3}$ \\
\hline$A_{i}$ & 0 & 1 & $3 \cdot 162$ & 1 & $3 \cdot 162$ & $4 \cdot 162$ & 0 & $0 \cdot 634$ \\
$\theta_{i}$ & 0 & 0 & 0 & 180 & 0 & 90 & 0 & $-97 \cdot 1$ \\
& $s_{1} s_{4}$ & $s_{1} s_{5}$ & $s_{2} s_{3}$ & $s_{2} s_{4}$ & $s_{2} s_{5}$ & $s_{3} s_{4}$ & $s_{3} s_{5}$ & $s_{4} s_{5}$ \\
$A_{i}$ & $2 \cdot 038$ & $1 \cdot 250$ & $2 \cdot 038$ & $0 \cdot 634$ & $0 \cdot 0$ & 0 & $0 \cdot 0$ & $0 \cdot 0$ \\
$\theta_{i}$ & $-112 \cdot 1$ & $-55 \cdot 5$ & $67 \cdot 9$ & $82 \cdot 9$ & $86 \cdot 6$ & 0 & $-86 \cdot 7$ & $-161 \cdot 5$ \\
& $s_{1} s_{2} s_{3}$ & $s_{1} s_{2} s_{4}$ & $s_{1} s_{2} s_{5}$ & $s_{1} s_{3} s_{4}$ & $s_{1} s_{3} s_{5}$ & $s_{1} s_{4} s_{5}$ & $s_{2} s_{3} s_{4}$ & $s_{2} s_{3} s_{5}$ \\
$A_{i}$ & 0 & 0 & 0 & 0 & $1 \cdot 209$ & $0 \cdot 483$ & 0 & $0 \cdot 483$ \\
$\theta_{i}$ & 0 & 0 & 0 & 0 & $-83 \cdot 9$ & $144 \cdot 0$ & 0 & $-36 \cdot 0$ \\
& $s_{2} s_{4} s_{5}$ & $s_{3} s_{4} s_{5}$ & $s_{1} s_{2} s_{3} s_{4}$ & $s_{1} s_{2} s_{3} s_{5}$ & $s_{1} s_{2} s_{4} s_{5}$ & $s_{1} s_{3} s_{4} s_{5}$ & $s_{2} s_{3} s_{4} s_{5}$ & $s_{1} s_{2} s_{3} s_{4} s_{5}$ \\
$A_{i}$ & $0 \cdot 968$ & 0 & 0 & $0 \cdot 0$ & $0 \cdot 0$ & $0 \cdot 0$ & $1 \cdot 250$ & 0 \\
$\theta_{i}$ & $-97 \cdot 6$ & 0 & 0 & $133 \cdot 7$ & $131 \cdot 2$ & $-50 \cdot 1$ & $124 \cdot 5$ & 0 \\
\hline
\end{tabular}

Table 4. The phase look-up table for Galileo E1 signals.

\begin{tabular}{lrrrrr}
\hline$s_{1}$ & $s_{2}$ & \multicolumn{1}{c}{$s_{3}$} & \multicolumn{1}{c}{$s_{5}$} & Phase angle (degrees) \\
\hline 1 & 1 & 1 & 1 & 1 & $17 \cdot 5$ \\
1 & 1 & 1 & 1 & -1 & $-17 \cdot 5$ \\
1 & 1 & -1 & -1 & 1 & $72 \cdot 5$ \\
1 & 1 & -1 & -1 & -1 & $-72 \cdot 5$ \\
1 & -1 & 1 & -1 & 1 & $162 \cdot 5$ \\
1 & -1 & 1 & -1 & -1 & $-162 \cdot 5$ \\
1 & -1 & -1 & 1 & 1 & $72 \cdot 5$ \\
1 & -1 & -1 & 1 & -1 & $-72 \cdot 5$ \\
-1 & 1 & 1 & -1 & 1 & $107 \cdot 5$ \\
-1 & 1 & 1 & -1 & -1 & $-107 \cdot 5$ \\
-1 & 1 & -1 & 1 & 1 & $17 \cdot 5$ \\
-1 & -1 & 1 & 1 & -1 & $-17 \cdot 5$ \\
-1 & -1 & 1 & 1 & 1 & $107 \cdot 5$ \\
-1 & -1 & -1 & -1 & -1 & $-107 \cdot 5$ \\
-1 & -1 & -1 & -1 & -1 & $162 \cdot 5$ \\
-1 & 1 & 1 & 1 & $-162 \cdot 5$ \\
\hline
\end{tabular}

\section{Since}

$$
\begin{gathered}
s_{1} s_{3}=s_{2} s_{4}=c_{E 1-D}(t) c_{E 1-P}(t), \\
s_{1} s_{4}=s_{2} s_{3}=c_{E 1-D}(t) c_{E 1-P}(t) s c_{B O C(1,1)} s c_{B O C(6,1)}, \\
s_{1} s_{5}=s_{2} s_{3} s_{4} s_{5}=c_{E 1-D}(t) s c_{B O C(1,1)} s_{P R S}(t), \\
s_{1} s_{3} s_{5}=s_{2} s_{4} s_{5}=c_{E 1-D}(t) c_{E 1-P}(t) s_{P R S}(t), s_{1} s_{4} s_{5}=s_{2} s_{3} s_{5}
\end{gathered}
$$

Equation (33) is simplified as

$$
\begin{aligned}
s(t)= & 3.317 c_{E 1-D}(t)\left(0.953 s c_{B O C(1,1)}(t)+0.302 s c_{B O C(6,1)}(t)\right) \\
& +3.317 c_{E 1-P}(t)\left(0.953 s c_{B O C(1,1)}(t)-0.302 s c_{B O C(6,1)}(t)\right) \\
& +3.317 j(0.953+0.302) s_{P R S}(t) \\
& -j 3.317(0.953-0.302) c_{E 1-D}(t) c_{E 1-P}(t) s_{P R S}(t)
\end{aligned}
$$


Table 5. The optimal phase values of POCET for Galileo E1 signals.

\begin{tabular}{|c|c|c|c|c|c|}
\hline$s_{1}$ & $s_{2}$ & $s_{3}$ & $s_{4}$ & $s_{5}$ & Phase angle (degrees \\
\hline 1 & 1 & 1 & 1 & 1 & 0 \\
\hline 1 & 1 & 1 & 1 & -1 & $322 \cdot 3$ \\
\hline 1 & 1 & 1 & -1 & 1 & $71 \cdot 2$ \\
\hline 1 & 1 & 1 & -1 & -1 & $251 \cdot 2$ \\
\hline 1 & 1 & -1 & 1 & 1 & $356 \cdot 6$ \\
\hline 1 & 1 & -1 & 1 & -1 & $325 \cdot 8$ \\
\hline 1 & 1 & -1 & -1 & 1 & $36 \cdot 4$ \\
\hline 1 & 1 & -1 & -1 & -1 & $286 \cdot 0$ \\
\hline 1 & -1 & 1 & 1 & 1 & $71 \cdot 2$ \\
\hline 1 & -1 & 1 & 1 & -1 & $251 \cdot 2$ \\
\hline 1 & -1 & 1 & -1 & 1 & $142 \cdot 3$ \\
\hline 1 & -1 & 1 & -1 & -1 & $180 \cdot 0$ \\
\hline 1 & -1 & -1 & 1 & 1 & $36 \cdot 4$ \\
\hline 1 & -1 & -1 & 1 & -1 & $286 \cdot 0$ \\
\hline 1 & -1 & -1 & -1 & 1 & $137 \cdot 1$ \\
\hline 1 & -1 & -1 & -1 & -1 & $185 \cdot 3$ \\
\hline-1 & 1 & 1 & 1 & 1 & $5 \cdot 3$ \\
\hline-1 & 1 & 1 & 1 & -1 & $317 \cdot 1$ \\
\hline-1 & 1 & 1 & -1 & 1 & $106 \cdot 0$ \\
\hline-1 & 1 & 1 & -1 & -1 & $216 \cdot 4$ \\
\hline-1 & 1 & -1 & 1 & 1 & $360 \cdot 0$ \\
\hline-1 & 1 & -1 & 1 & -1 & $322 \cdot 3$ \\
\hline-1 & 1 & -1 & -1 & 1 & $71 \cdot 2$ \\
\hline-1 & 1 & -1 & -1 & -1 & $251 \cdot 2$ \\
\hline-1 & -1 & 1 & 1 & 1 & $106 \cdot 0$ \\
\hline-1 & -1 & 1 & 1 & -1 & $216 \cdot 4$ \\
\hline-1 & -1 & 1 & -1 & 1 & $145 \cdot 8$ \\
\hline-1 & -1 & 1 & -1 & -1 & 176.6 \\
\hline-1 & -1 & -1 & 1 & 1 & $71 \cdot 2$ \\
\hline-1 & -1 & -1 & 1 & -1 & $251 \cdot 2$ \\
\hline-1 & -1 & -1 & -1 & 1 & $142 \cdot 3$ \\
\hline-1 & -1 & -1 & -1 & -1 & $180 \cdot 0$ \\
\hline
\end{tabular}

Considering that $\sqrt{10 / 11} \approx 0.953$, and $\sqrt{1 / 11} \approx 0.302$, Equation (35) is equivalent to Equation (28).

As a comparison, we also obtain the optimal phase values of POCET, which are listed in Table 5. The power constraints and phase constraints are given by Equation (30).

Following the method proposed by Zhang et al. (2012a), we translate the results of POCET into an analytical expression, i.e.

$$
\begin{aligned}
s_{\text {POCET }}(t)= & 0.1490 e^{-j 0.3287} s_{1}+0.4712 e^{-j 0.3287} s_{2}+0.1490 e^{j 2.8129} s_{3} \\
& +0.4712 e^{-j 0.3287} s_{4}+0.6203 e^{j 1.2421} s_{5}+0.0020 e^{-j 0.3287} s_{1} s_{2} s_{3} \\
& +0.1363 e^{j 2.8129} s_{1} s_{2} s_{4}+0.0178 e^{-j 1.8995} s_{1} s_{2} s_{5}+0.0020 e^{-j 0.3287} s_{1} s_{3} s_{4} \\
& +0.0411 e^{j 1.2421} s_{1} s_{3} s_{5}+0.0178 e^{-j 1.8995} s_{1} s_{4} S_{5}+0.1363 e^{-j 0.3287} s_{2} s_{3} s_{4} \\
& +0.0178 e^{-j 1.2421} s_{2} s_{3} s_{5}+0.2904 e^{-j 1.8995} s_{2} s_{4} s_{5}+0.0178 e^{j 1.2421} s_{3} s_{4} S_{5} \\
& +0.0482 e^{-j 1.8995} s_{1} s_{2} s_{3} s_{4} s_{5}
\end{aligned}
$$


Considering the relation between signal components, we have $s_{1} s_{2} s_{3}=s_{4}, s_{1} s_{2} s_{4}=s_{3}$, $s_{1} s_{3} s_{4}=s_{2}, s_{2} s_{3} s_{4}=s_{1}, s_{1} s_{2} s_{5}=s_{3} s_{4} s_{5}, s_{1} s_{3} s_{5}=s_{2} s_{4} s_{5}, s_{1} s_{4} s_{5}=s_{2} s_{3} s_{5}$ and $s_{1} s_{2} s_{3} s_{4} s_{5}=s_{5}$. Then Equation (36) is simplified as

$$
\begin{aligned}
s_{\text {POCET }}(t) & =0.2853 e^{-j 0.3287} s_{1}+0.4714 e^{-j 0.3287} s_{2}+0.2853 e^{j 2.8129} s_{3} \\
& +0.4714 e^{-j 0.3287} s_{4}+0.5721 e^{j 1.2421} s_{5}+0.5721 e^{j 1.2421} s_{1} s_{3} s_{5}
\end{aligned}
$$

We find that the power ratio of signal components is $1: 2 \cdot 7301: 1: 2 \cdot 7301: 4 \cdot 0211$, not the designed power ratio $1: 10: 1: 10: 17 \cdot 325$ ! Because the POCET method assumes that all the PRN code signals are uncorrelated, a single signal component is also unrelated with IM signals. In other words, Equation (1) is established only when all the PRN code signals are uncorrelated. However, some signal components are related in this case. The IM signals change the power of single signal components, which results in the failure of POCET.

5. THE APPLICATION OF THE SECOND WAY. In this section, we obtain the AltBOC-like modulation schemes with $85 \%$ and $86 \%$ combining efficiency by the second way. The baseband expression of the Galileo AltBOC signal is shown in Equation (1). $s_{1}$ and $s_{2}$ denote the data and pilot component at lower sideband, respectively. $s_{3}$ and $s_{4}$ denote the data and pilot component at upper sideband, respectively. $s c_{S}(t)$ and $s c_{P}(t)$ represent the four-valued subcarrier for the single signals and the product signals, respectively, whose waveforms in a period are illustrated in Figure 1 (Galileo OS SIS ICD, 2010). $T_{s}$ is the period of periodic side-band subcarrier functions.

$$
\begin{aligned}
s(t)= & \frac{1}{2 \sqrt{2}}\left(s_{1}+j s_{2}\right)\left[s c_{S}(t)-j s c_{S}\left(t-T_{s} / 4\right)\right] \\
& +\frac{1}{2 \sqrt{2}}\left(s_{3}+j s_{4}\right)\left[s c_{S}(t)+j s c_{S}\left(t-T_{S} / 4\right)\right] \\
& +\frac{1}{2 \sqrt{2}}\left(s_{2} s_{3} s_{4}+j s_{1} s_{3} s_{4}\right)\left[s c_{P}(t)-j s c_{P}\left(t-T_{S} / 4\right)\right] \\
& +\frac{1}{2 \sqrt{2}}\left(s_{1} s_{2} s_{4}+j s_{1} s_{2} s_{3}\right)\left[s c_{P}(t)+j s c_{P}\left(t-T_{S} / 4\right)\right]
\end{aligned}
$$

Figure 1 shows that each four-valued subcarrier period is sub-divided in eight equal sub-periods. In each sub-period, $s(t)$ can be seen as a constant envelope composite signal of four binary PRN code signals. The combining efficiency of AltBOC is $85 \cdot 36 \%$. Equation (38) is rewritten as (Lestarquit et al., 2008):

$$
\begin{aligned}
s(t)= & \frac{\sqrt{2+\sqrt{2}}}{4}\left(s_{1}+j s_{2}\right) e^{-j \pi\left(\frac{1}{8}+\frac{1}{4} k\right)}+\frac{\sqrt{2+\sqrt{2}}}{4}\left(s_{3}+j s_{4}\right) e^{j \pi\left(\frac{1}{8}+\frac{1}{4} k\right)} \\
& +\frac{\sqrt{2-\sqrt{2}}}{4}\left(s_{2} s_{3} s_{4}+j s_{1} s_{3} s_{4}\right) e^{-j \pi\left(\frac{5}{8}-\frac{3}{4} k\right)}+\frac{\sqrt{2-\sqrt{2}}}{4}\left(s_{1} s_{2} s_{4}+j s_{1} s_{2} s_{3}\right) e^{j \pi\left(\frac{5}{8}-\frac{3}{4} k\right)}
\end{aligned}
$$




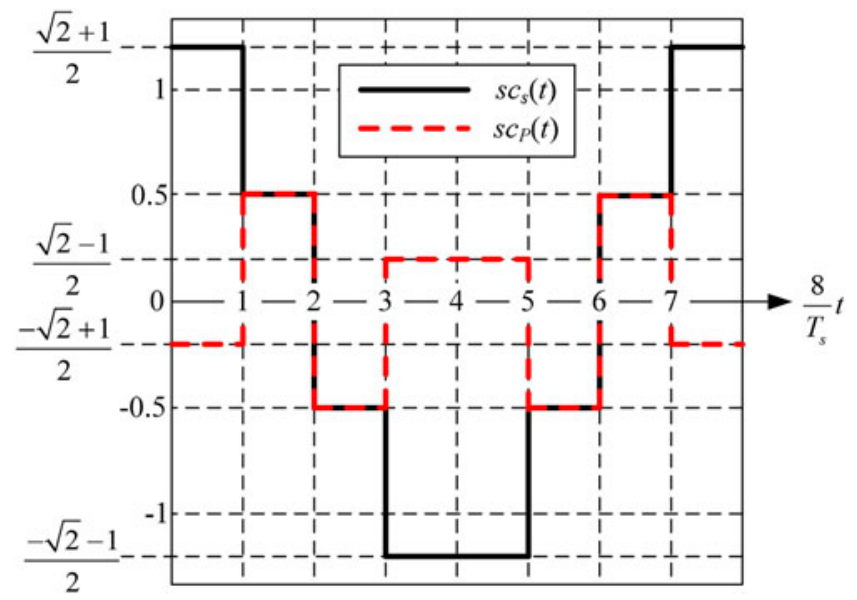

Figure 1. One period of the two subcarriers in AltBOC Modulation.

$k=0,1,2, \ldots, 7$ represents the eight sub-periods. In each sub-period, the power and phases of four signal components can been obtained by Equation (39). From the results of Zhang (2012b), we know that the combining efficiency of POCET is also $85 \cdot 36 \%$ when the power and phase constraints in Equation (39) are met.

Now we first begin to generate the AltBOC-like modulation with $85 \%$ combining efficiency. We exploit the objective function Equation (23). The combining efficiency is set to $\eta_{\text {set }}=85 \%$. In the first sub-period, we pre-set

$$
A_{1}=A_{2}=A_{3}=A_{4}=1, \theta_{1}=-\frac{1}{8} \pi, \theta_{3}=\frac{1}{8} \pi, \theta_{2}=\theta_{1}+90^{\circ}, \theta_{4}=\theta_{3}+90^{\circ}
$$

Then we obtain

$$
\begin{aligned}
s= & \left(s_{1}+j s_{2}\right) e^{-j \frac{\pi}{8}}+\left(s_{3}+j s_{4}\right) e^{j \frac{\pi}{8}}+0.4201\left(s_{2} s_{3} s_{4}+j s_{1} s_{3} s_{4}\right) e^{-j \pi \frac{123.9}{180}} \\
& +0.4201\left(s_{1} s_{2} s_{4}+j s_{1} s_{2} s_{3}\right) e^{j \pi \frac{101.1}{180}}
\end{aligned}
$$

In the second sub-period, we set $\theta_{1}=-\frac{3}{8} \pi, \theta_{3}=\frac{3}{8} \pi$, then we obtain

$$
\begin{aligned}
s= & \left(s_{1}+j s_{2}\right) e^{-j \frac{3 \pi}{8}}+\left(s_{3}+j s_{4}\right) e^{j \frac{3 \pi}{8}}+0.4201\left(s_{2} s_{3} s_{4}+j s_{1} s_{3} s_{4}\right) e^{j \pi \frac{11.1}{180}} \\
& +0.4201\left(s_{1} s_{2} s_{4}+j s_{1} s_{2} s_{3}\right) e^{-j \pi \frac{33.9}{180}}
\end{aligned}
$$


After we obtain all the expressions of the eight sub-periods, the AltBOC-like modulation with $85 \%$ combining efficiency is expressed as

$$
\begin{aligned}
s(t)= & \left(s_{1}+j s_{2}\right) e^{-j \pi\left(\frac{1}{8}+\frac{1}{4} k\right)}+\left(s_{3}+j s_{4}\right) e^{j \pi\left(\frac{1}{8}+\frac{1}{4} k\right)} \\
& +0.4201\left(s_{2} s_{3} s_{4}+j s_{1} s_{3} s_{4}\right) e^{-j \pi\left(\frac{123.9}{180}-\frac{3}{4} k\right)} \\
& +0.4201\left(s_{1} s_{2} s_{4}+j s_{1} s_{2} s_{3}\right) e^{j \pi\left(\frac{101.1}{180}-\frac{3}{4} k\right)}, t \bmod T_{s}=\left[\frac{k T_{s}}{8}, \frac{(k+1) T_{s}}{8}\right)
\end{aligned}
$$

Figure 2(a) shows the constellation diagram of AltBOC-like modulation with $85 \%$ combining efficiency. Its envelope value is constant, which is equal to $2 \cdot 1698$. Figure 2(b) shows the constellation diagram of AltBOC modulation with $85 \cdot 36 \%$ combining efficiency. We can see that there are only eight different phase values for AltBOC modulation. However, when the combining efficiency is reduced to $85 \%$, there are up to 24 different phase values for the AltBOC-like modulation. It appears that each point in Figure 2(b) splits into four points in Figure 2(a).

Next we begin to generate the AltBOC-like modulation with $86 \%$ combining efficiency. The objective function Equation (23) is used. In the first sub-period, the expression of the composite signal is expressed as

$$
\begin{aligned}
s=\left(s_{1}\right. & \left.+j s_{2}\right) e^{-j \frac{\pi}{4}}+\left(s_{3}+j s_{4}\right) e^{j \frac{\pi}{8}}+0.4035 e^{-j \frac{5}{8} \pi}\left(s_{2} s_{3} s_{4}+j s_{1} s_{3} s_{4}\right) \\
& +0.4035 e^{j \frac{5}{8} \pi}\left(s_{1} s_{2} s_{4}+j s_{1} s_{2} s_{3}\right)
\end{aligned}
$$

After we obtain all the expressions of the eight sub-periods, the AltBOC-like modulation with $86 \%$ combining efficiency is expressed as

$$
\begin{aligned}
s(t)= & \left(s_{1}+j s_{2}\right) e^{-j \pi\left(\frac{1}{8}+\frac{1}{4} k\right)}+\left(s_{3}+j s_{4}\right) e^{j \pi\left(\frac{1}{8}+\frac{1}{4} k\right)} \\
& +0.4035\left(s_{2} s_{3} s_{4}+j s_{1} s_{3} s_{4}\right) e^{-j \pi\left(\frac{5}{8}-\frac{3}{4} k\right)}+0.4035\left(s_{1} s_{2} s_{4}+j s_{1} s_{2} s_{3}\right) e^{j \pi\left(\frac{5}{8}-\frac{3}{4} k\right)}
\end{aligned}
$$

where $k=0,1, \ldots, 7$.

Figure 2(c) shows the constellation diagram of AltBOC-like modulation with $86 \%$ combining efficiency. Its envelope value is quasi-constant. There are eight different phase values and two different envelope values for this AltBOC-like modulation. The average envelope value is $2 \cdot 1567$. The maximum and minimum envelope values are $2 \cdot 1765$ and $2 \cdot 1368$ respectively. Compared with AltBOC modulation, it seems that every point in Figure 2(b) splits in the radial direction into two points in Figure 2(c).

Figure 3 depicts the normalized Auto-Correlation Functions (ACF) of the above three modulations in the case of $92.07 \mathrm{MHz}$ bandwidth. Figure 3(a) clearly shows that the ACFs of AltBOC-like modulations are similar to that of AltBOC modulation. With the increase of the combining efficiency, Figure 3(b) shows that the main-peak of the ACF becomes higher.

6. CONCLUSIONS. In this paper, a quasi-constant envelope multiplexing technique is proposed. Our method is more effective than the POCET method. The proposed method can work in two ways. The main advantages of our method are that the combining efficiency and IM signals are adjustable. When we slightly relax this 

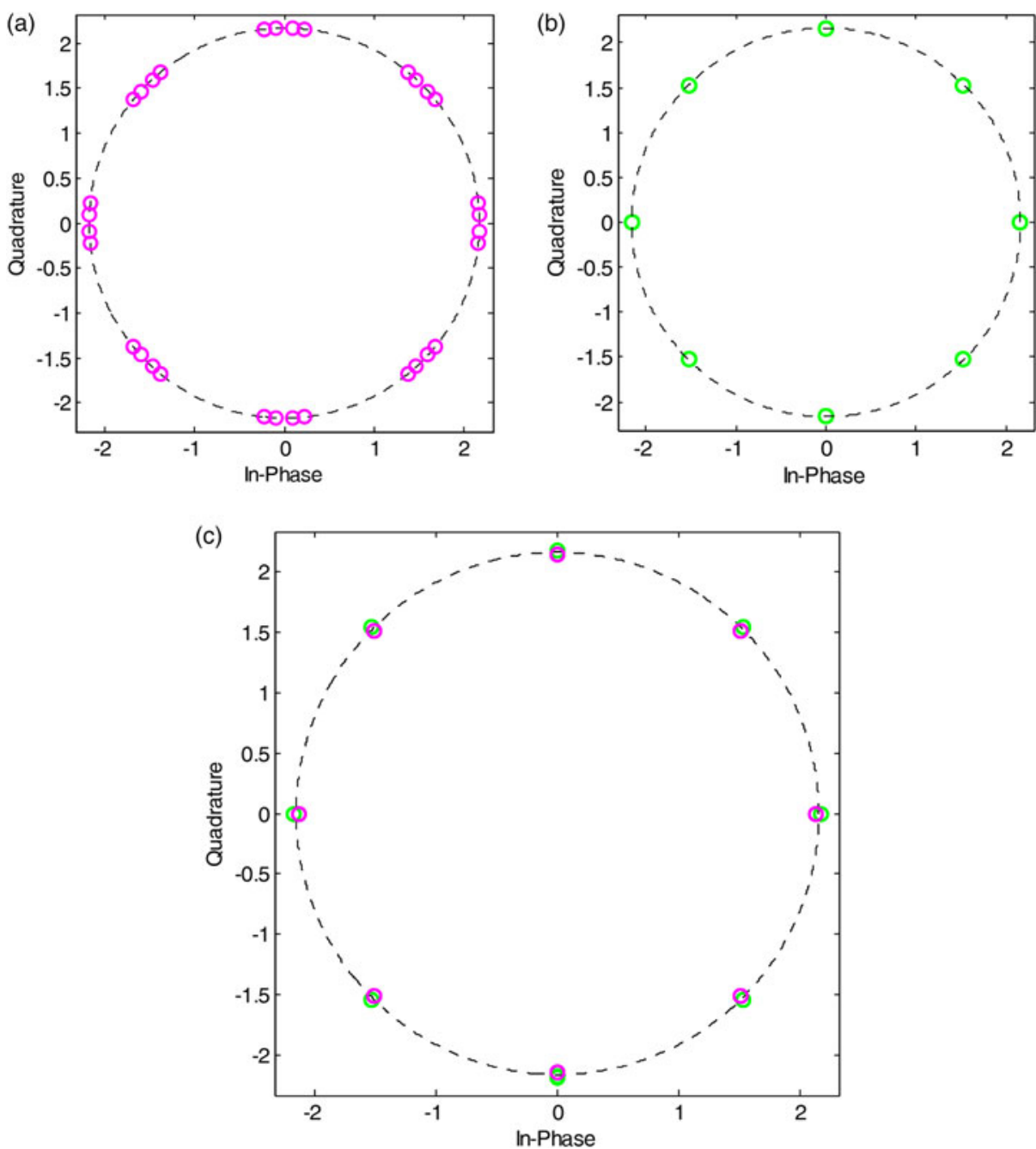

Figure 2. The constellation diagram. (a) AltBOC-like modulation with $85 \%$ combining efficiency.

(b) AltBOC modulation. (c) AltBOC-like modulation with $86 \%$ combining efficiency.

constraint of constant envelope, we can even achieve higher combining efficiency than POCET. The multiplexing applications of GPS L1 signals, Galileo E1 signals and AltBOC-like modulations verify the correctness and effectiveness of the proposed method. Based on the three examples, we summarise the following conclusions:

The example of GPS L1 signals shows that the first variation of our method is equivalent to POCET in terms of combining efficiency. The result also shows that for $N$ unrelated binary PRN signals, when the constant envelope constraint is strictly met, the POCET method does reach the highest combining efficiency.

The example of Galileo E1 signals shows our method can be applied to this case that some signal components are related to a certain extent. Due to the correlation between 

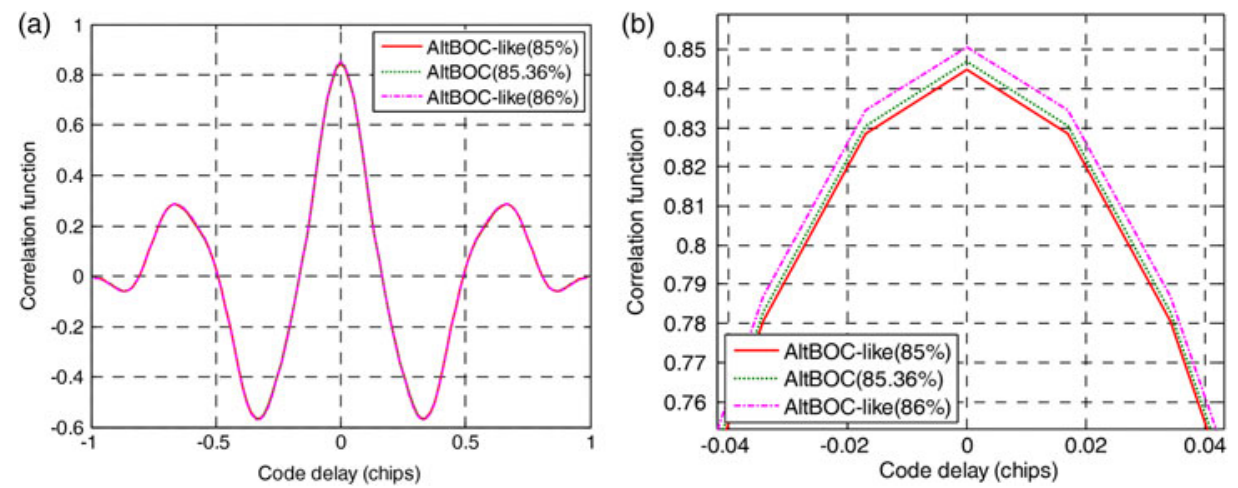

Figure 3. The auto-correlation function. (a) The full figure of ACF. (b) The enlarged view of the main-peak.

signal components, when POCET is used, the IM signals may change the power of single signal components, which results in the failure of POCET.

The last example shows that the second variation of our method is helpful to present more details of the combining. We can generate some AltBOC-like modulations with different combining efficiency. These AltBOC-like modulations have a similar function to AltBOC. Among them, AltBOC has the highest combining efficiency when keeping the envelope constant. Moreover, there are the least number of phase values in the constellation diagram of AltBOC. When we reduce the combining efficiency, the envelope is still constant. If the combining efficiency is higher than $85.36 \%$, the envelope becomes quasi-constant. Higher combining efficiency means a stronger main-peak in the ACF. However, the effect of quasi-constant envelope on signal performance should be further analysed before it is practically used.

\section{REFERENCES}

Butman, S. and Timor, U. (1972). Interplex-An Efficient Multichannel PSK/PM Telemetry System. IEEE Transaction on Communications, 20(3), 415-419.

Cahn, C.R. and Dafesh, P.A. (2011). Phase-optimized constant-envelope transmission (POCET) method, apparatus and system. U.S. Patent 0051783.

Dafesh, P.A. and Cahn, C.R. (2009). Phase-optimized constant-envelope transmission (POCET) modulation method for GNSS signals. Proceedings of the 22nd International Technical Meeting of The Satellite Division of the Institute of Navigation, Savannah, GA, 2860-2866.

Dafesh, P.A. and Cahn, C.R. (2011). Application of POCET method to combine GNSS signals at different carrier frequencies. Proceedings of the 24th International Technical Meeting of the Satellite Division of The Institute of Navigation, Porland OR, 1201-1206.

Dafesh, P.A., Nguyen, T.M. and Lazar, S. (1999). Coherent adaptive subcarrier modulation (CASM) for GPS modernization. Proceedings of ION National Technical Meeting, San Diego, CA, 649-660.

European GNSS (Galileo) Open Service Signal In Space Interface Control Document. (2010). OS SIS ICD, Issue 1.1,4-7.http://ec.europa.eu/enterprise/policies/satnav/galileo/files/galileo-os-sis-icd-issue1-revision1_en.pdf.

Fan, T., Lin, V.S., Wang, G.H. and Dafesh, P.A. (2008). Study of Signal Combining Methodologies for Future GPS Flexible Navigation Payload (Part II). Position, Location and Navigation Symposium, 2008 IEEE/ION, 1079-1089. 
Hein, G.W., Avila-Rodriguez, J.A., Ries, L., Lestarquit, L., Issler, J.L., Godet, J. and Pratt, T. (2005). A candidate for the Galileo L1 OS optimized signal. Proceedings of the 18th International Technical Meeting of the Satellite Division (ION GNSS 2005), Long Beach, CA, September 2005, 833-845.

Lestarquit, L., Artaud, G., and Issler, J.L. (2008). AltBOC for dummies or everything you always wanted to know about AltBOC. Proceedings of the 21st International Technical Meeting of the Satellite Division of The Institute of Navigation, Savannah, GA, 961-970.

Spilker Jr., J.J. and Orr, R.S. (1988). Code Multiplexing via Majority Logic for GPS Modernization. Proceedings of the 11th International Technical Meeting of the Satellite Division of The Institute of Navigation (ION GPS 1998), Nashville, TN, September 1998, 265-273.

Zhang, K, Zhou, H. and Wang, F. (2011). Multiplexing performance assessment of POCET method for Compass B1/B3 signals. The Journal of Navigation, 64, 41-54.

Zhang, K., Li, Y., Zhou, H. and Wang, F. (2012b). Analytical Transmission Model of POCET Technique for Compass B1 and B3 Signals. Proceedings of the 25th International Technical Meeting of the Satellite Division of The Institute of Navigation, Nashville, TN277-285.

Zhang, X.M., Zhang, X., Yao, Z. and Lu, M. (2012a). Implementation of Constant Envelope Multiplexing Based on Extended Interplex and Inter-Modulation Construction Method. Proceedings of the 25th International Technical Meeting of The Satellite Division of the Institute of Navigation (ION GNSS 2012), Nashville, TN, September 2012, 893-900. 